\title{
Adiponectin receptors gene expression and insulin sensitivity in non-diabetic Mexican Americans with or without a family history of Type 2 diabetes
}

\author{
A. E. Civitarese ${ }^{1}$ C. P. Jenkinson ${ }^{2}$ D. Richardson ${ }^{2}$ - M. Bajaj ${ }^{2}$ K. Cusi ${ }^{2}$ S. Kashyap ${ }^{2}$ R. Berria ${ }^{2}$. \\ R. Belfort ${ }^{2}$ - R. A. DeFronzo ${ }^{2}$ - L. J. Mandarino ${ }^{2}$ - E. Ravussin 1 \\ ${ }^{1}$ Pennington Biomedical Research Center, Baton Rouge, USA \\ ${ }^{2}$ Division of Diabetes, Department of Medicine, University of Texas Health Science Center, San Antonio, Texas, USA
}

\section{Abstract}

Aims/hypothesis. The recent discovery of two adiponectin receptors (AdipoR1 and AdipoR2) will improve our understanding of the molecular mechanisms underlying the insulin-sensitising effect of adiponectin. The aim of this study was to determine for the first time whether skeletal muscle AdipoR1 and/or AdipoR2 gene expression levels are associated with insulin resistance.

Methods. Using RT-PCR and northern analysis we measured AdipoRl and AdipoR2 gene expression in skeletal muscle from healthy Mexican Americans with normal glucose tolerance who had $(n=8)$ or did not have $(n=10)$ a family history of Type 2 diabetes.

Results. Gene expression profiling indicated that the AdipoR1 and AdipoR2 isoforms are highly expressed in human skeletal muscle, unlike in mice where Adi-
poR2 expression was highest in the liver, and AdipoR1 was highest in skeletal muscle. In the study subjects, the expression levels of AdipoRl $(p=0.004)$ and AdipoR2 $(p=0.04)$, as well as plasma adiponectin concentration $(p=0.03)$ were lower in people with a family history of Type 2 diabetes than in those with no family history of the disease. Importantly, the expression levels of both receptors correlated positively with insulin sensitivity ( $r=0.64, p=0.004$ and $r=0.47, p=0.048$ respectively).

Conclusions/interpretation. Collectively, these data indicate that both isoforms of the adiponectin receptor play a role in the insulin-sensitising effect of adiponectin.

Keywords Adiponectin - AdipoR1 - AdipoR2 - Insulin resistance $\cdot$ Mexican American · Type 2 diabetes
Received: 29 October 2003 / Accepted: 9 January 2004

Published online: 23 April 2004

(C) Springer-Verlag 2004

E. Ravussin (๘)

Pennington Biomedical Research Center, 6400 Perkins Rd, Baton Rouge, LA 70808 USA

E-mail: RavussE@pbrc.edu

Tel.: +1-225-763-3186, Fax: +1-225-763-3030

Abbreviations: AdipoR1, adiponectin receptor-1 . AdipoR2, adiponectin receptor- 2 - AMKP, adenosine 5 '-monophosphate-activated protein kinase $\cdot \mathrm{FH}+$, with a family history of Type 2 diabetes $\cdot$ FH-, with no family history of Type 2 diabetes - PPAR- $\alpha$, peroxisome proliferator-activated receptor- $\alpha$

\section{Introduction}

There is a growing list of proteins secreted by adipose tissue that have an impact on lipid and glucose metabolism and therefore insulin sensitivity and possibly energy balance. One such adipokine is adiponectin (also known as apm1, AdipoQ and Acrp30), which is exclusively expressed in white adipose tissue [1]. While many other adipokines are associated with insulin resistance, adiponectin appears to be unique in that it has insulin-sensitising effects. Adiponectin expression is lower in rodent models of insulin resistance [2] and obese diabetic humans [3, 4]. In fact, several studies have found positive associations between adiponectin mRNA and/or plasma concentration and insulin sensitivity [3,5]. More importantly, a high plasma concentration of adiponectin is protective 
against the development of Type 2 diabetes in Pima Indians [6].

Recently, it was demonstrated that adiponectin directly activates adenosine $5^{\prime}$-monophosphate-activated protein kinase $(A M P K)$, thereby stimulating glucose uptake in vivo [7]. In the same set of experiments, adiponectin increased fatty acid oxidation in cultured myocytes, whereas in the liver, the expression of key gluconeogenic enzymes was reduced [7]. However, the identity of the cellular receptor(s) for adiponectin remained elusive until the recent cloning and characterisation of two isoforms of the gene, adiponectin receptor-1 (AdipoRl) and adiponectin receptor-2 (AdipoR2) [8]. In mice, AdipoRl was abundantly expressed in skeletal muscle, whereas AdipoR2 was predominantly expressed in the liver. By interfering with RNA expression of both receptors, it was shown that adiponectin-induced activation of $A M P K$ was blunted, causing an impairment of fatty acid oxidation and glucose uptake [8]. This suggests that a down-regulation of the $R 1$ and $R 2$ receptors in skeletal muscle may be involved in the development of insulin resistance.

The present study was undertaken to determine for the first time whether skeletal muscle AdipoRl and/or AdipoR2 gene expression levels are associated with insulin resistance in a population of subjects enriched in insulin resistance genes. Using RT-PCR, we measured AdipoRl and AdipoR2 gene expression in the skeletal muscle of healthy, normal-glucose-tolerant Mexican Americans with or without a strong family history of Type 2 diabetes.

\section{Subjects and methods}

Subjects. Studies were conducted in the General Clinical Research Center of the South Texas Veterans Health Care System, Audie Murphy Division, in San Antonio, USA. Eight healthy normoglycaemic subjects with a family history of Type 2 diabetes (FH+) and ten control subjects with no family history of Type 2 diabetes (FH-) were recruited. The presence of a family history was ascertained by asking the subjects whether they had at least two known first-degree relatives with Type 2 diabetes. Groups were matched for age and body composition. Subjects did not exercise regularly and all were instructed not to exercise within 48 hours of the study. All subjects were Mexican Americans and all had normal glucose tolerance as assessed by a 75-g oral glucose tolerance test. Hyperinsulinaemic euglycaemic clamps $\left(40 \mathrm{mU} \cdot \mathrm{m}^{-2} \cdot \mathrm{min}^{-1}\right)$ with infusion of $3-{ }^{3} \mathrm{H}$-glucose for isotopic measurement of glucose disposal ( $\mathrm{M}$ value) and percutaneous vastus lateralis muscle biopsies were performed as previously described [9]. Body composition was determined by bioimpedance (RJLBio, Detroit, Mich., USA). Plasma glucose concentration was measured on a Beckman glucose analyser (Fullerton, Calif., USA). Plasma adiponectin (Linco Research, St. Louis, Mo., USA) and insulin (Diagnostics Products, Los Angeles, Calif., USA) concentrations were measured by radioimmunoassay. Plasma NEFA concentrations were assayed by an enzymatic method (NEFA-C kit; Wako Pure Chemicals, Osaka, Japan). The Institutional Review Board of the University of Texas Health Science Cen- ter at San Antonio approved the research and all subjects gave written informed consent.

Real-time quantitative RT-PCR. Total RNA was isolated using Trizol (Invitrogen, Carlsbad, Calif., USA), and concentration was determined spectrophotometrically at 260/280 nm. AdipoRl and AdipoR2 transcripts were quantitated using real-time RT-PCR technology on a BioRad I Cycler (BioRad, Hercules, Calif., USA). Primer sequences were as follows: AdipoRl forward primer 5'-TTCTTCCTCATGGCTGTGATGT-3', AdipoR1 reverse primer 5'-AAGAAGCGCTCAGGAATTCG -3'; AdipoR 1 probe $5^{\prime}$-FAM-TCACTGGAGCTGGCCTTTATGCTGC-BHQ1-3'. AdipoR2 forward primer 5'-ATAGGGCAGATAGGCTGGTTGA-3', AdipoR2 reverse primer 5'-GGATCCGGGCAGCATACA-3'; AdipoR2 probe $5^{\prime}$-FAM-TGATGGCCAGCCTCTACATCACAGGA-3'. PCR conditions were $48{ }^{\circ} \mathrm{C}$ for $30 \mathrm{~min}$ and $95{ }^{\circ} \mathrm{C}$ for $10 \mathrm{~min}$, followed by 40 cycles of $95{ }^{\circ} \mathrm{C}$ for $15 \mathrm{~s}$ and $60{ }^{\circ} \mathrm{C}$ for $1 \mathrm{~min}$. AdipoRl and AdipoR2 mRNA content was normalised to cyclophilin expression.

Northern blot analysis. Human multiple northern blot filters (BioBiosciences, Austin, Tex., USA) were hybridised with [ $\alpha-$ $\left.{ }^{32} \mathrm{P}\right] \mathrm{dATP}\left(10^{6} \mathrm{cpm} / \mathrm{ml}\right)$-labelled AdipoRl or AdipoR2 human skeletal muscle cDNA in ULTRAhyb at $42{ }^{\circ} \mathrm{C}$ for $24 \mathrm{~h}$. The filters were washed in $2 \times \mathrm{SSC}, 0.1 \% \mathrm{SDS}$ at $42{ }^{\circ} \mathrm{C}$.

Statistical analysis. All reported values are means \pm SEM. Differences between groups were determined using an independent $t$ test. Since all the data were normally distributed, Pearson correlation coefficients were calculated to determine the relationship between selected variables, and multiple linear regression models were used to examine these relationships after adjusting for covariates such as sex. Differences were considered significant when $p$ values were less than 0.05 .

\section{Results}

Fasting plasma insulin concentrations were significantly higher in $\mathrm{FH}+$ subjects than in $\mathrm{FH}-$ subjects $(p=0.02$ ), whereas $\mathrm{M}$ values (glucose disposal rate during the euglycaemic clamp) were significantly lower in $\mathrm{FH}+$ subjects than in $\mathrm{FH}-$ subjects $(p=0.001$; Table 1). There was no difference in plasma adiponec-

Table 1. Differences in the characteristics of subjects with and without a family history of Type 2 diabetes

\begin{tabular}{lccl}
\hline Characteristic & $\mathrm{FH}+$ & $\mathrm{FH}-$ & $p$ value \\
\hline Sex (male/female) & $3 / 5$ & $6 / 4$ & \\
Age (years) & $36 \pm 3$ & $37 \pm 4$ & $\mathrm{NS}$ \\
BMI $\left(\mathrm{kg} / \mathrm{m}^{2}\right)$ & $28 \pm 1$ & $28 \pm 2$ & $\mathrm{NS}$ \\
Body fat $(\%)$ & $34 \pm 3$ & $29 \pm 3$ & $\mathrm{NS}$ \\
Fasting glucose $(\mathrm{mmol} / \mathrm{l})$ & $51 \pm 2$ & $53 \pm 2$ & $\mathrm{NS}$ \\
Fasting insulin $(\mu \mathrm{U} / \mathrm{ml})$ & $10 \pm 1$ & $6 \pm 1$ & 0.02 \\
Non-esterified fatty acids & $0.58 \pm 0.05$ & $0.57 \pm 0.05$ & $\mathrm{NS}$ \\
$\quad(\mathrm{mmol} / \mathrm{l})$ & & & \\
M value $\left(\mathrm{mg}^{-1} \mathrm{~kg}^{-1} \cdot \mathrm{min}^{-1}\right)$ & $3.0 \pm 0.3$ & $5.6 \pm 0.5$ & 0.001 \\
Plasma adiponectin $(\mu \mathrm{g} / \mathrm{ml})$ & $5.4 \pm 0.4$ & $8.8 \pm 1.3$ & 0.03
\end{tabular}

Data are means $\pm \mathrm{SE}$; $\mathrm{FH}+$, subjects with family history of diabetes; $\mathrm{FH}-$, subjects with no family history of diabetes; M value, rate of insulin-stimulated glucose disposal during a hyperinsulinaemic euglycaemic clamp 


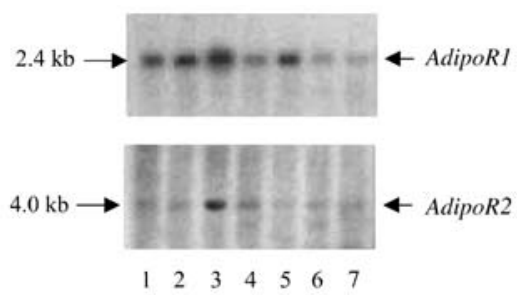

b

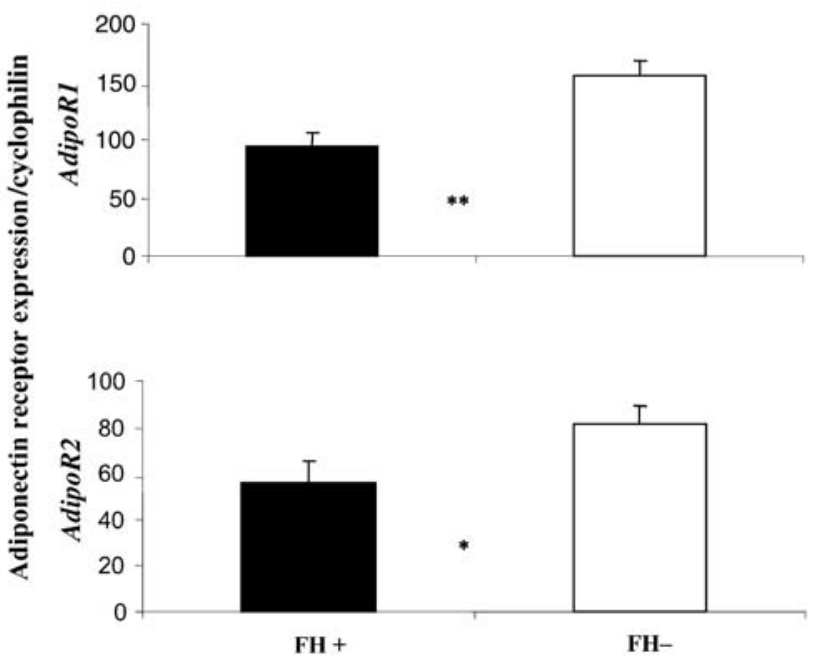

Fig. 1. a Northern blot analysis of adiponectin receptor-1 (AdipoR1, top panel) and adiponectin receptor-2 (AdipoR2, bottom panel) mRNA in human tissues. Lanes: 1, brain; 2, placenta; 3, skeletal muscle; 4, heart; 5, kidney; 6, pancreas; 7, liver. b Skeletal muscle gene expression of AdipoRl and AdipoR2 in subjects with at least two first-degree relatives with Type 2 diabetes $(\mathrm{FH}+)$ and ten control subjects with no family history of Type 2 diabetes (FH-). ${ }^{*} p=0.04, * * p=0.004$

tin concentrations between male and female subjects. Plasma adiponectin concentrations were lower in $\mathrm{FH}+$ subjects than in $\mathrm{FH}-$ subjects $(5.4 \pm 0.41$ vs $8.9 \pm 1.3$, $p=0.03)$ and correlated positively with $\mathrm{M}$ values ( $r=0.52, p=0.03$; data not shown).

Using a commercially available human mRNA library (BD Biosciences, San Jose, Calif., USA) we detected AdipoRl gene expression in the adrenals, liver, cerebellum, whole brain, thyroid, colon, bone marrow, testis, uterus and trachea, but the highest expression was in skeletal muscle. Similarly, the AdipoR2 gene was ubiquitously expressed, with highest expression in the testis, muscle, liver and adipose tissue (data not shown). Northern blotting of human tissues (Fig. 1a) identified a major single band of $2.4 \mathrm{~kb}$ for AdipoRl and $4.0 \mathrm{~kb}$ for AdipoR2. Both receptor isoforms were predominantly expressed in human skeletal muscle.

Skeletal muscle AdipoRl gene expression was lower in FH+ subjects than in FH- subjects $(94 \pm 12.3$ vs $155 \pm 12.7, p=0.004$; Fig. 1b) and was positively correlated with glucose disposal rate (M value; $r=0.64$, $p=0.004$; Fig. 2, top). Similarly, skeletal muscle AdipoR2 gene expression was lower in $\mathrm{FH}+$ subjects than

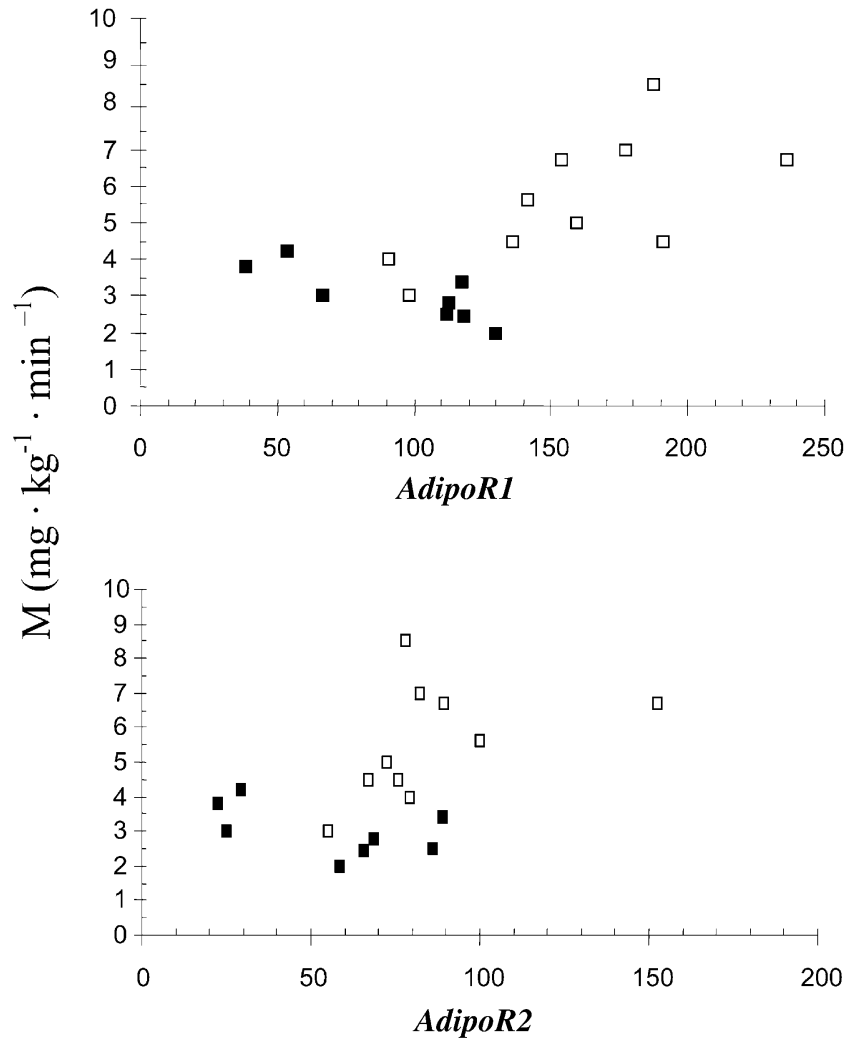

\section{Adiponectin receptor expression/cyclophilin}

Fig. 2. Insulin sensitivity assessed by euglycaemic hyperinsulinaemic clamp (M value, $\mathrm{mg} \cdot \mathrm{kg}^{-1} \cdot \mathrm{min}^{-1}$ ) vs adiponectin receptor-1 (AdipoR1; $r=0.64, p=0.004$ ) and adiponectin receptor-2 (AdipoR2; $r=0.47, p=0.048$ ) gene expression levels in skeletal muscle. M, glucose disposal rate; black squares, subjects with a family history of Type 2 diabetes; white squares, subjects with no family history of Type 2 diabetes

in $\mathrm{FH}-$ subjects $(56 \pm 9$ vs $81 \pm 8, p=0.04$; Fig. $1 \mathrm{~b})$ and correlated positively with insulin sensitivity $(r=0.47$, $p=0.048$; Fig. 2, bottom) and plasma adiponectin concentration $(r=0.59, p=0.01$; data not shown).

\section{Discussion}

The results of the present study show that AdipoRI and AdipoR2 are highly expressed in human skeletal muscle, re-emphasising the importance of this tissue for the potential insulin-sensitising effect of adiponectin in humans. Yamauchi et al. (supplementary info; Fig. 1d) also found that AdipoRl and AdipoR2 were both expressed in human skeletal muscle [8]. However, the AdipoR2 receptor was more abundant in mouse liver and AdipoRl mRNA expression was higher in cultured mouse myocytes, suggesting that AdipoRl may be the predominant form in mouse skeletal muscle [8]. In contrast, our results suggest that both AdipoR1 and AdipoR2 are highly expressed in human skeletal muscle. A definitive answer on the relative abundance of $R 1$ and $R 2$ proteins cannot be pro- 
vided at this time since antibodies to these receptors are not yet available.

Our preliminary data indicate that there is a strong relationship between insulin sensitivity and adiponectin receptor expression in humans. Both AdipoRl and AdipoR2 expression levels were significantly lower in normal-glucose-tolerant individuals with a strong family history of Type 2 diabetes (i.e. at high risk of developing the disease) than in volunteers with no family history. The impaired expression of the receptors, in combination with lower concentrations of the circulating hormone, may be part of the phenotype predisposing this group to the development of the disease. We have previously shown such individuals to be characterised by profound insulin resistance, with abnormalities in insulin receptor signalling in human muscle $[9,10]$. As expected, the individuals with a family history of diabetes were insulin resistant, with increased fasting plasma insulin concentrations, decreased glucose disposal rates during insulin infusion and decreased plasma adiponectin levels. Importantly, subjects with a family history of diabetes were matched with control subjects (without family history) for body fat, another confounding factor when comparing plasma levels of adiponectin and/or insulin sensitivity. As previously reported [3,5], we confirmed that plasma adiponectin concentrations were positively correlated with insulin sensitivity measured by the hyperinsulinaemic clamp, further supporting the notion that adiponectin is an insulin-sensitising hormone. More importantly, we report for the first time that insulin-stimulated glucose disposal correlates positively with the expression level of both isoforms of the adiponectin receptor, suggesting that they play a role in skeletal muscle insulin resistance. In addition, and surprisingly, AdipoR2 expression positively correlated with plasma adiponectin concentrations. Without knowledge of the molecular structure of both adiponectin receptor isoforms it is impossible to predict how the expression of these receptors is regulated in human tissues. Only when the entire promoter sequence is described will we understand the impact of transcription factors and physiological milieu on the regulation of the receptors. Studies clarifying these interactions are now underway.

Yamauchi et al. proposed that the AdipoR1 receptor was the predominant isoform in muscle, through which adiponectin activated $A M P K$ (and peroxisome proliferator-activated receptor- $\alpha[P P A R-\alpha])$, thereby increasing carnitine palmitoyltranferase- 1 activity in cultured $\mathrm{C} 2 \mathrm{C} 12$ myocytes [8]. We propose here that both isoforms of the adiponectin receptor may mediate adiponectin action in human muscle for the following reasons: (i) AdipoRI and AdipoR2 are primarily expressed in skeletal muscle; (ii) both isoforms are expressed at lower levels in individuals prone to diabetes and (iii) insulin sensitivity is positively correlated with the expression of both receptor isoforms. A down-regulation in the level of receptor expression may reduce PPAR- $\alpha$ and $A M P K$ activation by adiponectin, thereby reducing fatty acid oxidation and favouring the accumulation of triglycerides within skeletal muscle. This mechanism is consistent with the effect of adiponectin to decrease muscle triglyceride content and consequently improve muscle insulin sensitivity $[2,11]$.

Full-length adiponectin is cleaved proteolytically to release the $\mathrm{C}$-terminal globular domain. Although both full-length and globular adiponectin bind to AdipoR1 and AdipoR2 receptors and probably initiate downstream events, the two receptors appear to have different binding affinities for these two ligands [7, 8]. In vitro binding assays in mouse $\mathrm{C} 2 \mathrm{C} 12$ myocytes demonstrate a high affinity of the AdipoRl receptor for globular adiponectin and a low affinity for the fulllength molecule, whereas the AdipoR2 receptor has a lower affinity for both the globular and full-length adiponectin [8]. However, given the reported differences in gene sequence between murine and human receptors, and the species differences in the level of receptor expression, further work is required to investigate the binding and efficacy of globular and fulllength adiponectin to the AdipoRl and AdipoR2 isoforms in human skeletal muscle.

In summary, our results demonstrate that both forms of the adiponectin receptor are expressed in skeletal muscle. The expression of both isoforms is lower in normal-glucose-tolerant Mexican Americans with a strong family history of Type 2 diabetes mellitus than in control subjects, and is positively correlated with insulin sensitivity. Both AdipoRl and AdipoR2 isoforms may be important therapeutic targets for improving insulin sensitivity in individuals with Type 2 diabetes or in people at risk of developing the disease.

Acknowledgements. Funding for this work was provided by the grants NIDDKR01-DK60412 (E. Ravussin), NIHR01DK-47936 (L. J. Mandarino) and NIHR01DK-24092 (R. A. DeFronzo), a VA Merit Award (R. A. DeFronzo) and a General Clinical Research Center Grant (M01-RR-01346). The authors acknowledge the nursing assistance of John Kincaid, Jim King, Norma Diaz and Patricia Wolfe, as well as the expert technical assistance of Jean Finlayson, Sheila Taylor, Cindy Munoz, Kathy Camp and Richard Castillo. We also acknowledge Dr George Argyropoulos for providing us with the human mRNA library and Dr Leonie Heilbronn for her critical comments on this work.

\section{References}

1. Maeda K, Okubo K, Shimomura I, Funahashi T, Matsuzawa Y, Matsubara K (1996) cDNA cloning and expression of a novel adipose specific collagen-like factor, apM1 (Adipose Most abundant Gene transcript 1). Biochem Biophys Res Commun 221:286-289

2. Yamauchi T, Kamon J, Waki H et al. (2001) The fat-derived hormone adiponectin reverses insulin resistance associated with both lipoatrophy and obesity. Nat Med 7:941-946 
3. Kern PA, Di Gregorio GB, Lu T, Rassouli N, Ranganathan G (2003) Adiponectin expression from human adipose tissue: relation to obesity, insulin resistance, and tumor necrosis factor-alpha expression. Diabetes 52:1779-1785

4. English PJ, Coughlin SR, Hayden K, Malik IA, Wilding JP (2003) Plasma adiponectin increases postprandially in obese, but not in lean, subjects. Obes Res 11:839-844

5. Weyer C, Funahashi T, Tanaka S et al. (2001) Close association with insulin resistance and hyperinsulinemia. J Clin Endocrinol Metab 86:1930-1935

6. Lindsay RS, Funahashi T, Hanson RL et al. (2002) Adiponectin and development of type 2 diabetes in the Pima Indian population. Lancet 360:57-58

7. Yamauchi T, Kamon J, Minokoshi Y et al. (2002) Adiponectin stimulates glucose utilization and fatty-acid oxidation by activating AMP-activated protein kinase. Nat Med 8:1288-1295
8. Yamauchi T, Kamon J, Ito Y et al. (2003) Cloning of adiponectin receptors that mediate antidiabetic metabolic effects. Nature 423:762-769

9. Pratipanawatr W, Pratipanawatr T, Cusi K et al. (2001) Skeletal muscle insulin resistance in normoglycemic subjects with a strong family history of type 2 diabetes is associated with decreased insulin-stimulated insulin receptor substrate1 tyrosine phosphorylation. Diabetes 50:2572-2578

10. Gulli G, Rossetti L, DeFronzo RA (1997) Hyperamylinemia is associated with hyperinsulinemia in the glucose-tolerant, insulin-resistant offspring of two Mexican-American non-insulin-dependent diabetic parents. Metabolism 46:1157-1161

11. Fruebis J, Tsao TS, Javorschi S et al. (2001) Proteolytic cleavage product of $30-\mathrm{kDa}$ adipocyte complement-related protein increases fatty acid oxidation in muscle and causes weight loss in mice. Proc Natl Acad Sci USA 98:20052010 